\title{
Detection of lymph node micrometastases in malignant mammary tumours in dogs by cytokeratin immunostaining
}

\author{
A. J. F. Matos, A. M. R. Faustino, C. Lopes, G. R. Rutteman, F. Gärtner
}

\begin{abstract}
A series of 131 local and regional lymph nodes from $\mathbf{4 0}$ dogs with malignant mammary tumours were evaluated by staining with haematoxylin and eosin and immunohistochemically for antibodies to pancytokeratin (AE1/AE3) and cytokeratin 14. The immunohistochemical tests detected occult micrometastases in 9-2 per cent of the lymph nodes that were negative by haematoxylin and eosin staining. Under the modified TNM classification of canine mammary tumours, these results raised the clinical stage of 12.5 per cent of the affected dogs. However, if the latest TNM classification of human breast cancer had been applied, none of the animals would have been reclassified.
\end{abstract}

IN 1980 the first edition of a TNM classification for tumours ( $\mathrm{T}$ relates to the size of the primary tumour, $\mathrm{N}$ corresponds to the presence [or absence] of lymph node metastasis, and $\mathrm{M}$ relates to the detection [or not] of distant metastasis), including those of the mammary glands of dogs, was published by the World Health Organization (WHO) (Owen 1980). The authors stated that 'The TNM system . . . will require modification in the future?. Few studies have assessed the prognostic value of either the 1980 system or a modification of the TNM clinical classification of mammary tumours of dogs (Rutteman and others 2001). This modified classification, like that for human breast cancer, uses the size of the primary tumour, the status of the regional lymph nodes (assessed by cytology or histology of biopsies) and the detection of distant metastases as the main criteria for the staging of affected patients (Rutteman and others 2001, Sobin and Wittekind 2002). In human patients the use of screening mammography has improved the detection of small breast tumours (Singletary and Greene 2003), and led to the creation of more extensive categories in the staging system of the TNM classification for human breast cancer, including the status of intramammary lymph nodes. In addition immunohistochemistry and the reverse transcription-PCR are now used to detect cancer cells (Sobin and Wittekind 2002). The definition of a micrometastasis is based on size. Whereas the prognostic significance of $\mathrm{N}$-status, as determined by classical histology, has been well-established for several decades in human breast cancer (Nemoto and others 1980), the clinical significance of micrometastases is still unclear (Cserni and others 2003, Gusterson 2003). Immunostaining is a valuable tool for the detection of micrometastases in lymph nodes in which the results of sections stained with haematoxylin and eosin are negative or doubtful (Wong and others 2001, Ishida and others 2002).

In dogs, the modified TNM classification states that all N1 cases (animals with histological or cytological evidence of metastases) are classified as either stage III (any T, N1, M0) or stage IV (any T, any N, M1) (Rutteman and others 2001). The classification does not take into account differences between the location of positive nodes, for example, intramammary or supramammary, and ipsi- or contralateral, or between the size of the metastatic deposits, the relevance of isolated or small clusters of neoplastic cells, or of the methods used to detect occult micrometastases. The clinical value of the status of regional lymph nodes in canine mammary tumours is a subject of debate; some authors consider that metastases are a prognostic factor (Hellmén and others 1993, Yamagami and others 1996) but others consider that there is no significant relationship between their presence and the survival or disease-free interval for dogs (Schneider and others 1969, Nieto and others 2000). To the authors' knowledge, there are no reports on the prognostic value of occult lymph node micrometastases in dogs with malignant mammary tumours.

More small mammary tumours are being detected and surgically removed from dogs by veterinary practitioners. Their awareness that the type of surgery is not a major prognostic factor, provided that histologically adequate resection is achieved (MacEwen and others 1985), has led veterinary surgeons to choose nodulectomy or partial mastectomy as the correct procedure for small masses. As a result, pathologists often have no access to the regional lymph nodes, and have to decide the dog's nodal status on the basis of the local or intramammary nodes alone.

In human pathology, because only about 1 per cent of the lymph node tissue removed is evaluated by histopathological analysis the extent of metastatic disease may be underestimated (Wong and others 2001). Immunohistochemical techniques are a valuable method for the complete assessment of lymph nodes and the identification of occult micrometastases (Wong and others 2001). The aim of this study was to determine the value of immunohistochemistry in the detection of occult nodal micrometastases in dogs with malignant mammary tumours.

\section{MATERIALS AND METHODS}

Tissue samples

Sixty-two malignant mammary tumours and 131 local and regional lymph nodes were surgically removed from 40 female dogs aged between five and 14 years (mean 10.5 years) of various pure or mixed breeds. The tissues were preserved in 10 per cent buffered formalin, dehydrated and embedded in paraffin wax. Sections $4 \mu \mathrm{m}$ thick were cut and stained with haematoxylin and eosin for histological examination, and sections $3 \mu \mathrm{m}$ thick were used for immunohistochemical examination.

Histological examination The tumours were classified independently by two of the authors (F. G. and A. F.) on sections stained with haematoxylin and eosin, by using the diagnostic criteria proposed by the WHO classification of tumours in domestic animals (Misdorp and others 1999), and the lymph nodes were classified according to whether they contained cancer cells. When there was initially disagreement, a consensus diagnosis was reached by using a multihead microscope.

Immunohistochemistry Lymph node sections adjacent to those stained with haematoxylin and eosin were immunostained by using the modified avidin-biotin-peroxidase complex method (Hsu and others 1981). The following primary
Veterinary Record (2006) $158,626-630$

A. J. F. Matos, DVM, A. M. R. Faustino, $\mathrm{PhD}$, C. Lopes,

Instituto de Ciências Biomédicas de Abel Salazar, University of Porto, Largo Professor Abel Salazar, 2, 4099-003 Porto, Portugal

G. R. Rutteman, PhD, Clinic of Companion Animals, Utrecht University, PO Box 80125, 3508 TC Utrecht, The Netherlands F. Gärtner, PhD, Institute of Molecular Pathology and Immunology of the University of Porto, Rua Dr Roberts Frias s/n, 4200-465 Porto, Portugal

Correspondence to Dr Gärtner 


\begin{tabular}{|c|c|c|c|}
\hline \multicolumn{4}{|c|}{$\begin{array}{l}\text { TABLE 1: Evaluation of lymph node micrometastases in canine } \\
\text { malignant mammary tumours by staining with haematoxylin } \\
\text { and eosin (H and E) or by cytokeratin immunohistochemistry } \\
\text { (IHC) }\end{array}$} \\
\hline Nodal status & $\begin{array}{c}\text { Dogs }(\%) \\
(n=40)\end{array}$ & $\begin{array}{c}\text { Tumours (\%) } \\
(\mathrm{n}=62)\end{array}$ & $\begin{array}{l}\text { Lymph nodes }(\%) \\
(n=131)\end{array}$ \\
\hline $\mathrm{H}$ and $\mathrm{E}+$ & $7(17.5)$ & $8(13)$ & $12(9 \cdot 2)$ \\
\hline $\mathrm{IHC}+$ & $7(17.5)$ & $8(13)$ & $11(8.4)$ \\
\hline IHC - & 0 & 0 & $1(0.8)$ \\
\hline $\mathrm{H}$ and $\mathrm{E}-$ & 33 (82.5) & $54(87)$ & $119(90 \cdot 8)$ \\
\hline $\mathrm{IHC}+$ & $5(12.5)$ & $10(16)$ & $11(8.4)$ \\
\hline $\mathrm{IHC}-$ & $28(70)$ & 44 (71) & $108(82 \cdot 4)$ \\
\hline
\end{tabular}

antibodies were used: anti-pancytokeratin antibody AE1/AE3 (Zymed Laboratories) diluted 1:50, and anti-cytokeratin 14 (clone LL002; Serotec Laboratories) diluted 1:10. In the antipancytokeratin antibody AE1/AE3, the AE1 reacts against cytokeratins $10,14,15$ and 16 of the acidic subfamily, and the AE3 recognises the entire basic cytokeratin subfamily. This antibody cocktail allows all cells of epithelial origin to be visualised. The anti-cytokeratin 14 antibody is used to identify basal/myoepithelial cells (Vos and others 1993a, b). Tissue sections $3 \mu \mathrm{m}$ thick were cut from the formalin-fixed material and deparaffinised; the antigens were recovered by immersing the sections in target-retrieval solution (Dako) diluted $1: 10$, for 30 minutes at $100^{\circ} \mathrm{C}$ in a water bath. The slides were cooled in buffer for 20 minutes at room temperature and rinsed twice in phosphate-buffered saline for five minutes. Endogenous peroxidase activity was blocked by treating them with 3 per cent hydrogen peroxide in methanol for 10 minutes. Non-specific staining was eliminated by incubating the sections with normal rabbit serum (Dako) diluted 1:5 in a wet chamber for 20 minutes at room temperature. Excess serum was removed and the sections were incubated with the specific primary antibody overnight at $4^{\circ} \mathrm{C}$ in a wet chamber. They were then incubated with a 1:200 dilution of biotin-labelled anti-mouse secondary antibody (Dakopatts) for 30 minutes, followed by incubation with the avidin-biotin complex (Dakopatts) diluted 1:100 for 30 minutes. They were rinsed carefully with phosphate-buffered saline between each step of the procedure. The colour was developed with a freshly prepared solution of 3,3'-diaminobenzidine for up to seven minutes at room temperature, and the sections were lightly counterstained with haematoxylin, dehydrated and mounted. Negative controls for the immunostaining were made by substituting the primary antibody with a mouse immunoglobulin G1 antibody-clone Dak-Gol (Dakopatts). Positive controls were made from sections of canine mammary tumours known to express AE1/AE3 and cytokeratin 14.

\section{Evaluation of immunohistochemical data}

The immunohistochemically stained slides were evaluated by the same two authors without any knowledge of the haematoxylin and eosin results. The size of the nodal micrometastases was measured by using the computer-assisted stereological toolbox (CAST-grid, version 1.6; Olympus). The dogs were considered to be 'upstaged' if metastatic disease was found in any of the immunohistochemically stained nodes previously classified as negative on the slides stained with haematoxylin and eosin.

\section{RESULTS}

The rates of detection of micrometastases in the tumours and lymph nodes stained with haematoxylin and eosin and by cytokeratin immunohistochemistry are compared in Table 1 .

\section{Dogs}

Six of the 40 dogs were large breed, 21 were medium breed and 13 were small breed dogs; 21 were mixed-breed dogs, and there were seven poodles, three cocker spaniels, three boxers, two pointers, one Siberian husky, one fox terrier, one basset hound and one Pekinese. Thirty-seven of them were intact and three had been ovariohysterectomised. When the dogs were examined their regional lymph nodes were all considered normal. Thirteen of the dogs had more than one malignant tumour (from two to five distinct tumours) and 26 also had benign mammary tumours (from one to eight distinct tumours). The results of the evaluations of the lymph node micrometastases by the two staining techniques agreed in 35 ( 87.5 per cent) of the dogs, seven being positive in both evaluations and 28 negative in both.

\section{Tumours}

Sixty-two malignant tumours were removed from the 40 dogs. Three tumours were classified as carcinoma in situ, 12 as complex carcinomas, 31 as simple carcinomas (15 tubulopapillary, 15 solid and one anaplastic), seven as special carcinomas (five spindle cell carcinomas and two mucinous carcinomas), five as carcinosarcomas and four as carcinomas in a benign tumour. Forty-two of the tumours were located in the inguinal and caudal abdominal glands (M5 and M4), 12 in the cranial abdominal gland (M3) and eight in the thoracic glands (M1 and M2). The results of the evaluations of the lymph node micrometastases by the two staining techniques agreed in 52 (84 per cent) of the tumours, eight being positive in both evaluations and 44 negative in both Discrepancies were observed in lymph nodes draining from 10 (18.5 per cent) of the 54 tumours that were classified N0 on the slides stained with haematoxylin and eosin (four complex carcinomas, three simple carcinomas and three carcinosarcomas).

\section{Lymph nodes}

Between one and 15 local and regional lymph nodes, on average three, were evaluated from each dog. Immunohistochemistry confirmed the presence of metastases in 11 of the 12 nodes that were positive by haematoxylin and eosin. The results of the two techniques agreed in 119 (91 per cent) of the 131 nodes, 11 ( 8.4 per cent) being positive in both evaluations and 108 being negative in both. Eleven ( $9 \cdot 2$ per cent) of the 119 nodes that were negative by haematoxylin and eosin were positive by immunohistochemistry for cytokeratins. Of these 11 nodes, only two had cells that were immunoreactive for cytokeratin 14, and 10 had cells that were positive for the antibody against pancytokeratin AE1/AE3. In one node, staining with haematoxylin and eosin detected only metastatic invasion in lymphatic vessels around the node, whereas the immunohistochemical staining revealed intranodal deposits of cancer cells. There was one micrometastasis in two of the nodes, and more than one in the other nine. In three nodes, the micrometastases measured $2 \mathrm{~mm}$ but in the others they were less than $2 \mathrm{~mm}$; they ranged in size from $0 \cdot 1$ to $2 \mathrm{~mm}$ with a mean of $0.6 \mathrm{~mm}$.

In seven of the nodes, the metastatic cells appeared isolated and scattered (Figs 1,2). In one node, draining from a tubulopapillary carcinoma, isolated metastatic cells invaded the entire node in a disorganised pattern. In the remaining 14 positive nodes there were clusters of metastatic cells, ranging from disorganised clusters to tubular and papillary structures (Figs 3, 4).

\section{DISCUSSION}

The search for prognostic factors in human breast cancer has been a major subject of research for several decades (Cserni 


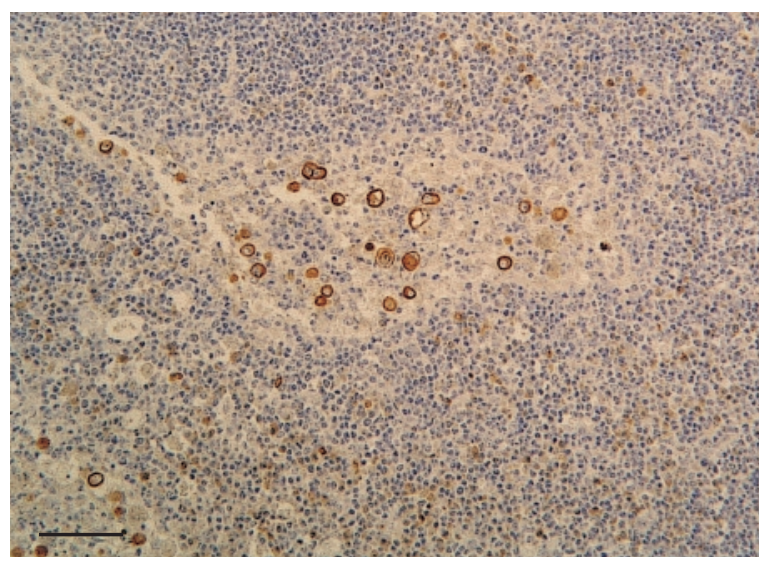

FIG 1: Lymph node showing isolated cancer cells from an anaplastic carcinoma, immunostained for cytokeratin AE1/AE3. Bar $=100 \mu \mathrm{m}$

and others 2003). The status of axillary lymph nodes is considered critical for the assessment of the outcome and the response of a cancer to treatment (Harris and others 1993, Hayes 1994). In dogs with mammary tumours the prognostic value of the status of their lymph nodes is a subject of debate. In some studies (Schneider and others 1969, Nieto and others 2000) it is considered to be of no value, but in others (Hellmén and others 1993, Yamagami and others 1996) it is considered to be significant. One multivariate analysis indicated that, after weighting for the mode of growth (infiltration), the lymph node status did not have additional prognostic value (Misdorp and Hart 1976). Possible reasons for these discrepancies are differences in the methods used to prepare and stain the tissue samples, in the number of nodes studied, in the follow-up time, in the detection of metastases and in the statistical analysis. In spite of clear definitions in human pathology of what must be considered as lymph node micrometastases (measurements between $0.2 \mathrm{~mm}$ and $2 \mathrm{~mm}$ in their greatest dimension) and clandestine metastases (the presence of tumour emboli in the subcapsular marginal sinuses of a lymph node) (Friedman and others 1988, Sobin and Wittekind 2002) there are still doubts about the proper TNM classification of some node categories (Gusterson 2003). In fact, although isolated tumour cells or metastatic deposits less than $0.2 \mathrm{~mm}$ should be considered as N0, it is not clear why this should be the minimum size limit. There is also no agreement about the number of sections that should be made of a node, a fact that may allow the assumption that a

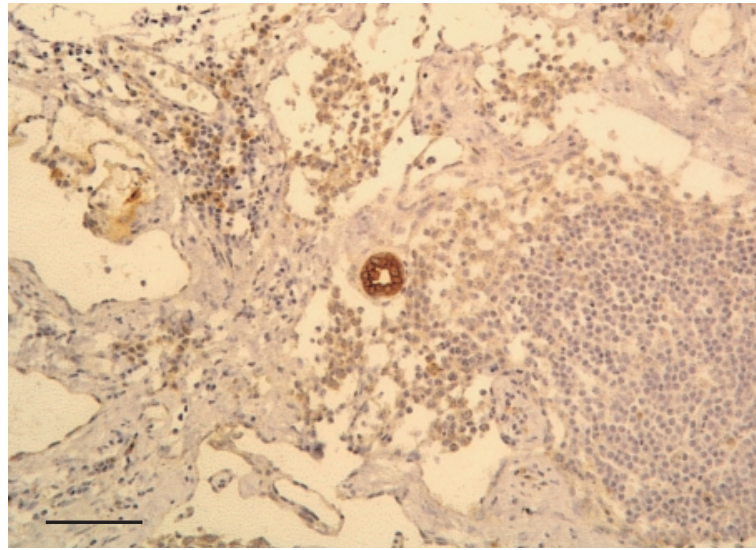

FIG 3: Lymph node showing a small cluster of cancer cells in a tubular arrangement from a complex carcinoma immunostained for cytokeratin AE1/AE3. Bar $=100 \mu \mathrm{m}$

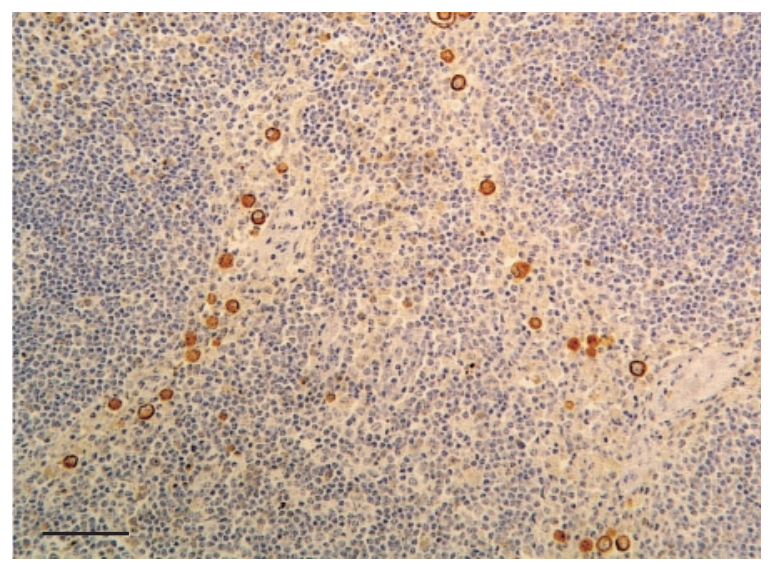

FIG 2: Lymph node showing isolated cancer cells from an anaplastic carcinoma immunostained for cytokeratin AE1/AE3. Bar $=100 \mu \mathrm{m}$

micrometastasis of $0.1 \mathrm{~mm}$ in one section may be part of a much larger one in another section of the same node.

There are some similarities between breast cancer in women and mammary tumours in bitches, but the differences may be sufficient to justify a different approach to lymph nodes metastases and their importance. In dogs, the lymph nodes linked anatomically to the mammary glands are in two distinct regions (axillary and inguinal), in contrast with only the axillary region in women; the presence of one mammary gland (cranial abdominal) often draining to both regions is also a major difference. The fact that some dogs are affected simultaneously by different histological types of malignant tumours is a further potential source of problems in the adaptation of the human criteria. The lack of knowledge of the total number of intramammary nodes that may be present in the mammary chain and to what extent the presence of a tumour may affect their number, location or draining pathways (Pereira and others 2003), is also an obstacle to the adaptation of the human criteria (that emphasise the number and location of the affected nodes) to dogs.

In dogs, as in women, the TNM classification uses the status of the lymph nodes for the clinical staging of mammary tumours. Although the human classification considers various groups in the $\mathrm{N}$ (regional lymph nodes) classification (Sobin and Wittekind 2002), the modified staging for dogs (Rutteman and others 2001) considers only N0 (no histological or cytological metastases) and N1 (histological or cytological metastases). The oversimplification of this system is obvious and hampers the study of the clinical relevance of micrometastases in bitches. The mere distinction between N0 and N1, without reference to the size of the metastatic deposits, may 'upstage' a dog affected by a small malignant tumour, from stage I (N0) directly to stage IV, simply because some isolated cells are found in one of the lymph nodes exam-

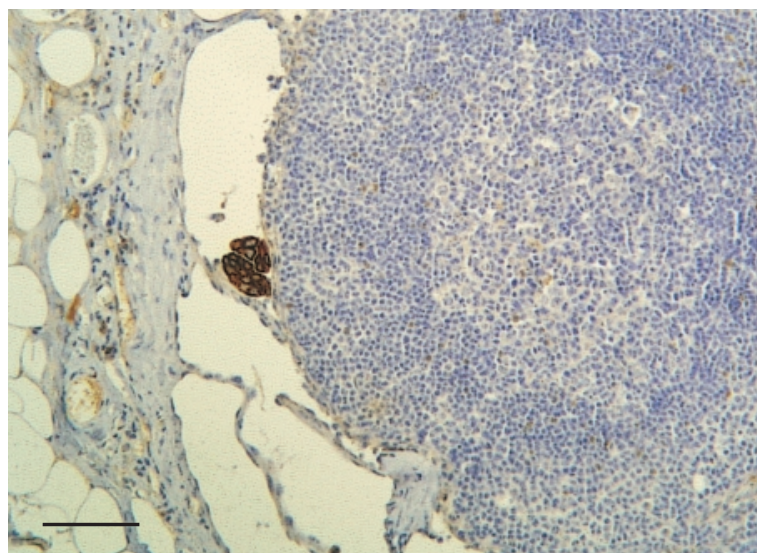

FIG 4: Lymph node showing a small cluster of disorganised cancer cells in the subcapsular sinus from a carcinosarcoma immunostained for cytokeratin AE1/AE3. Bar $=100 \mu \mathrm{m}$ 
ined. On the other hand, if recent human criteria are used, all the nodes in the same animal may have metastatic clusters of cancer cells less than $0.2 \mathrm{~mm}$ in size, but the dog will still be considered in stage I. If further therapeutic actions are to be based on the animal's clinical stage, more detailed criteria for the status of each animal are required. However, the more categories that are assessed for their prognostic weight the larger the series that needs to be studied, and such studies are difficult to conduct in veterinary oncology. Multicentre studies using standardised processing methods should overcome this problem and reach valid conclusions.

The use of immunohistochemistry for cytokeratin, although more expensive and technically more laborious, is, in the authors' opinion, a good way to deal with cases in which micrometastases are not detectable by routine haematoxylin and eosin evaluation (occult micrometastases). It makes clear the distinction between cancer cells and the normal cellular population of the lymph nodes, easing the task of the observer and providing a definite diagnosis. The technique has been used in studies of sentinel lymph nodes in many women with breast cancer, and resulted in the upstaging of between 3 and 47 per cent of them, depending on the precise techniques applied (Cserni and others 2003).

In this study, $9 \cdot 2$ per cent of the lymph nodes that were considered unaffected when evaluated with haematoxylin and eosin revealed micrometastatic deposits when examined immunohistochemically. These deposits may have been overlooked because they were only isolated cells, or because of the lack of organisation of very small clusters or because haemorrhage within the nodes masked the cancer cell deposits. It is also possible that there were no cancer cells in a section stained with haematoxylin and eosin, but their presence in adjacent sections stained positively by immunohistochemistry.

The detection of occult micrometastases resulted in the upstaging of 18.5 per cent of the tumours in the 40 dogs. Where they were detected, all the metastases stained positively with AE1/AE3, regardless of the tumour's histological classification, but only two nodes contained cancer cells that stained with CK14. This fact suggests that epithelial cells may be more prone to early metastasis than myoepithelial cells.

The diagnosis of more than one malignant tumour in two dogs in which occult micrometastases were discovered, three in one dog and four in the other, makes it difficult to attribute the metastatic deposits to a specific tumour, so all the malignancies were considered in these cases.

Five of the dogs were upstaged as a result of the detection of occult micrometastases in the sections stained immunohistochemically. According to the modified canine TNM classification for mammary tumours, two dogs in stage III, one in stage II and two in stage I, were reclassified to stage IV. However, if the latest criteria for the TNM classification of human breast cancer had been applied (Singletary and Greene 2003), none of them would have been reclassified, because the occult micrometastases were either isolated cells and clusters no larger than $0 \cdot 2 \mathrm{~mm}(\mathrm{~N} 0)$, or clusters no larger than $2 \mathrm{~mm}(\mathrm{~N} 1 \mathrm{mi})$.

There are conflicting results concerning the clinical relevance of occult micrometastases in the outcome of individual women with breast cancer, both in terms of overall survival and disease-free survival (Cserni and others 2003). To the authors' knowledge, there have been no studies of their relevance in dogs.

\section{References}

CSERNI, G., AMENDOEIRA, I., APOSTOLIKAS, N., BELLOCQ, J. P., BIANCHI, S., BUSSOLATI, G., BOECKER, W., BORISCH, B., CONNOLLY, C. E., DECKER, T., DERVAN, P., DRIJKONINGEN, M., ELLIS, I. O., ELSTON, C. W., EUSEBI, V., FAVERLY, D., HEIKKILA, P., HOLLAND, R., KERNER, H., KULKA, J., JACQUEMIER, J., LACERDA, M., MARTINEZ-PENUELA, J., DE
MIGUEL, C., PETERSE, J. L., RANK, F., REGITNIG, P., REINER, A., SAPINO, A., SIGAL-ZAFRANI, B., TANOUS, A. M., THORSTENSON, S., ZOZAYA E. \& WELLS, C. A. (2003) Pathological work-up of sentinel lymph nodes in breast cancer. Review of current data to be considered for the formulation of guidelines. European Journal of Cancer 39, 1654-1667

FRIEDMAN, S., BERTIN, F., MOURIESSE, H., BENCHABAT, A., GENIN, J., SARRAZIN, D. \& CONTESSO, G. (1988) Importance of tumour cells in axillary node sinus margins (clandestine metastasis) discovered by serial sectioning in operable breast carcinoma. Acta Oncologica 27, 483-487

GUSTERSON, B. A. (2003) The new TNM classification and micrometastases. The Breast 12, 387-390

HARRIS, J. R., MORROW, M. \& BONADONNA, G. (1993) Cancer of the breast. In Cancer: Principles \& Practice of Oncology. 4th edn. Eds V. T. DeVita, S Hellman, S. A. Rosenberg. Philadelphia, J. B. Lippincott. pp 1264-1332

HAYES, D. F. (1994) Tumour markers for breast cancer. Current utilities and future prospects. Hematology/Oncology Clinics of North America 8, 485-506 HELlMÉN, E., BERGSTRÖM, R., HOLMBERG, L., SPÅNGBERG, I-B. HANSSON, K. \& LINDGREN, A. (1993) Prognostic factors in canine mammary tumors: a multivariate study of 202 consecutive cases. Veterinary Pathology 30, 20-27

HSU, S. M., FAINE, L. \& FANGER, H. (1981) A comparative study of the peroxidase-antiperoxidase method and the avidin-biotin complex method for studying polypeptide hormones with radioimmunoassay antibodies American Journal of Clinical Pathology 75, 734-738

ISHIDA, M., KITAMURA, K., KINOSHITA, J., SASAKI, M., KUWAHARA, H. \& SUGIMACHI, K. (2002) Detection of micrometastases in the sentinel lymph nodes in breast cancer. Surgery 131,211-216

MACEWEN, E. G., HARVEY, H. J., PATNAIK, A. K., MOONEY, S., HAYES, A., KURZMAN, I. \& HARDY, W. D. (1985) Evaluation of effects of levamisole and surgery on canine mammary cancer. Journal of Biological Response Modifiers 4, 418-426

MISDORP, W., ELSE, R. W., HELLMÉN, E. \& LIPSCOMB, T. P. (1999) Histological classification of the mammary tumours of the dog and the cat. In World Health Organization International Histological Classification of Tumours of Domestic Animals. Second Series. Vol 7. Ed F. I. Shulman. Washington DC, AFIP. pp 1-59

MISDORP, W. \& HART, A. A. (1976) Prognostic factors in canine mammary cancer. Journal of the National Cancer Institute 56, 779-786

NEMOTO, T., VANA, J., BEDWANI, R. N., BAKER, H. W., MCGREGOR, F. H. \& MURPHY, G. P. (1980) Management and survival of female breast cancer. Results from a national survey by the American College of Surgeons. Cancer 45, 2917-2924

NIETO, A. I., PEÑA, L., PÉREZ-ALENZA, M. D., SÁNCHEZ, M. A., FLORES, J. M. \& CASTAÑO, M. (2000) Immunohistologic detection of estrogen receptor alpha in canine mammary tumours: clinical and pathologic associations and prognostic significance. Veterinary Pathology 37, 239-247

OWEN, L. N. (1980) TNM Classification of Tumors in Domestic Animals. Ed VPH/CMO/80.20. Geneva, World Health Organization

PEREIRA, C. T., RAHAL, S. C., DE CARVALHO BALIEIRO, J. C. \& RIBEIRO, A. A. (2003) Lymphatic drainage on healthy and neoplastic mammary glands in female dogs: can it really be altered? Anatomia, Histologia, Embryologia 32, 282-290

RUTTEMAN, G. R., WITHROW, S. J. \& MACEWEN, E. G. (2001) Tumors of the mammary gland. In Small Animal Oncology. 3rd edn. Eds S. J. Withrow, E. G. MacEwen. St Louis, W. B. Saunders. pp 455-477

SCHNEIDER, R., DORN, C. R. \& TAYLOR, D. O. N. (1969) Factors influenc ing canine mammary cancer development and postsurgical survival. Journal of the National Cancer Institute 43, 1249-1261

SINGLETARY, S. E. \& GREENE, F. L. (2003) Revision of breast cancer staging: the 6th edition of the TNM classification. Seminars in Surgical Oncology 21, 53-59

SOBIN, L. H. \& WITTEKIND, CH. (2002) TNM Classification of Malignant Tumours. 6th edn. New York, UICC, Wiley-Liss

VOS, J. H., VAN DEN INGH, T. S., MISDORP, W., MOLENBEEK, R. F., VAN MIL, F. N., RUTTEMAN, G. R., IVANYI, D. \& RAMAEKERS, F. C. (1993a Immunohistochemistry with keratin, vimentin, desmin, and alpha-smooth muscle actin monoclonal antibodies in canine mammary gland: malignant mammary tumours. Veterinary Quarterly 15, 96-102

VOS, J. H., VAN DEN INGH, T. S., MISDORP, W., MOLENBEEK, R. F., VAN MIL, F. N., RUTTEMAN, G. R., IVANYI, D. \& RAMAEKERS, F. C. (1993b) Immunohistochemistry with keratin, vimentin, desmin, and alpha-smooth muscle actin monoclonal antibodies in canine mammary gland: normal mammary tissue. Veterinary Quarterly 15, 102-107

WONG, S. L., CHAO, C., EDWARDS, M. J., SIMPSON, D. \& MCMASTERS,

K. M. (2001) The use of cytokeratin staining in sentinel lymph node biopsy for breast cancer. American Journal of Surgery 182, 330-334

YAMAGAMI, T., KOBAYASHI, T., TAKAHASHI, K. \& SUGIYAMA, M. (1996) Prognosis for canine malignant tumours based on TNM and histologic classification. Journal of Veterinary Medical Sciences 58, 1079-1083 